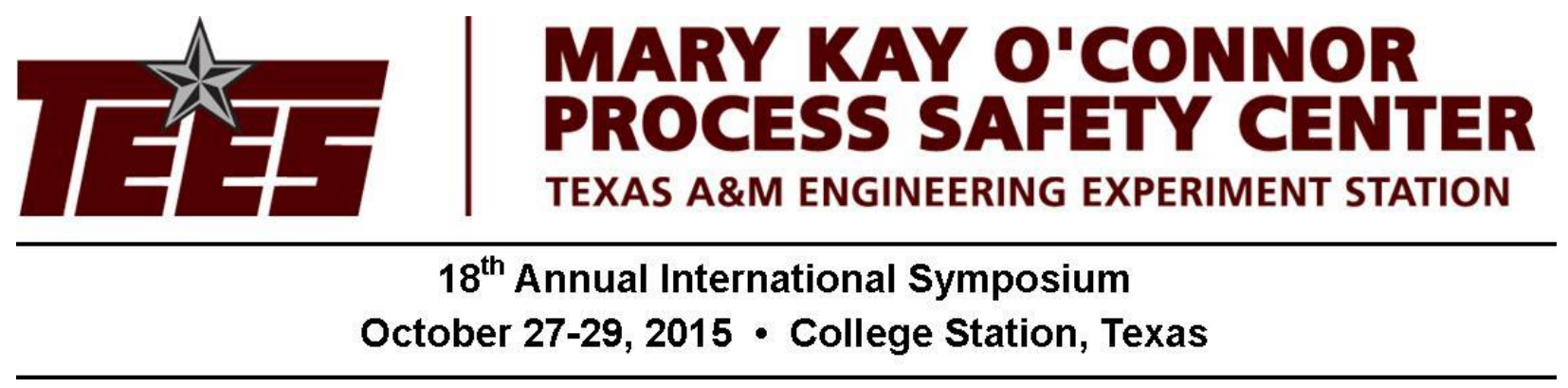

\title{
A Study of the Blast Wave Shape from Elongated VCEs
}

\author{
Jihui Geng, J. Kelly Thomas and Quentin Baker* \\ Baker Engineering and Risk Consultants (BakerRisk) \\ 3330 Oakwell Court, Suite 100 \\ San Antonio, TX 78218-3024 \\ (210) 824-5960 \\ * Presenter E-mail: QBaker@BakerRisk.com
}

\begin{abstract}
Elongated congestion patterns are common at chemical processing and petroleum refining facilities due to the arrangement of processing units. The accidental vapor cloud explosion (VCE) which occurred at the Buncefield, UK facility involved an elongated congested volume formed by the trees and undergrowth along the site boundary. Although elongated congested volumes are common, there have been few evaluations reported for the blast loads produced by elongated VCEs. Standard VCE blast load prediction techniques do not directly consider the impact of this congested volume geometry versus a more compact geometry.

This paper discusses an evaluation performed to characterize the blast loads from elongated VCEs and identified some significant differences in the resulting blast wave shape versus those predicted by well-known VCE blast load methodologies (e.g., BST and TNO MEM). The standard blast curves are based on an assumption that the portion of the flammable gas cloud participating in the VCE is hemispherical and located at grade level. The results of this evaluation showed that the blast wave shape for a deflagration in an elongated congested volume is similar to that for an acoustic wave in the near-field along the long-axis direction. Like an acoustic wave, an elongated VCE blast wave has a very quick transition from the positive phase peak pressure to the negative phase peak pressure, relative to the positive phase duration. The magnitude of the applied negative pressure on a building face depends strongly on the transition time between the positive and negative phase peak pressures, and this applied negative phase can be important to structural response under certain conditions. The main purpose of this evaluation was to extend previous work in order to investigate how an elongated VCE geometry impacts the resultant blast wave shape in the near-field. The influence of the normalized flame travel distance and the flame speed on the blast wave shape is also examined.
\end{abstract}




\section{Introduction}

Elongated congestion patterns are commonly seen at most chemical processing facilities. The accidental VCE which occurred at the Buncefield, UK facility involved an elongated congested volume. It was concluded ${ }^{1}$ that the trees and undergrowth along a plant boundary on the Buncefield site, which formed an elongated congested volume, caused flame acceleration up to a velocity of several hundred $\mathrm{m} / \mathrm{s}$. This elongated congestion played a key role in the flame acceleration, resulting blast loads and subsequent observed structural damage. In particular, the blast loads from the Buncefield incident were reported to show overpressures up to about 2 barg at the cloud/congestion edge, which then diminished rapidly with distance.

Blast waves from explosion sources like a VCE, pressure vessel burst or high explosive exhibit both positive and negative phases, and the relative magnitude of the positive and negative phases varies among these types of explosion sources and the specific source characteristics ${ }^{2}$. VCEs can be categorized into two modes, deflagrations and detonations, according to propagation mechanisms. In a vapor cloud deflagration, the flame propagates through the unburned fuel-air mixture at a flame velocity less than the speed of sound in the reactant gas mixture, while a detonation propagates at supersonic velocity. The blast curves for simplified VCE blast load prediction methodologies are normally based on an assumption that the flammable gas cloud is hemispherical and located at grade level ${ }^{3}$. Numerous studies ${ }^{4,5,6,7}$ have shown that as the flame speed increases, the rate of pressure rise and the peak blast overpressure both increase. VCE blast waves can be characterized in three regimes ${ }^{8}$ : acoustic wave, pressure wave and shock wave. These blast wave regimes can be overlaid on Baker-Strehlow-Tang (BST) scaled overpressure curves ${ }^{9}$. The approximate bounds of regimes in terms of flame speed are as shown in Table 1.

Table 1. Blast Wave Shape Regimes

\begin{tabular}{|c|c|}
\hline Blast Wave Regime & Approximate Flame Speed Range $\left(\mathbf{M}_{\mathbf{f}}\right)$ \\
\hline Acoustic & $<0.35$ \\
\hline Pressure Wave & $0.35<\mathrm{M}_{\mathrm{f}}<1.0$ \\
\hline Shock Wave & $>1.0$ \\
\hline
\end{tabular}

Although elongated congested volumes are common, there have been few evaluations reported for the blast loads produced by elongated VCEs ${ }^{10}$ and the blast loads from the elongated VCEs have not been fully addressed in VCE prediction methods. The authors recently performed a evaluation ${ }^{11}$ to characterize elongated VCEs and to identify the resultant difference in blast wave shape compared to those predicted by well-known VCE blast prediction methodologies. BakerRisk's Blast Wave Target Interaction (BWTI ${ }^{\mathrm{TM}}$ ) computational fluid dynamics (CFD) $\operatorname{code}^{12,13}$ was used to investigate the VCE blast loads resulting from elongated flammable gas clouds. The BWTITM predictions ${ }^{11}$ were validated against test data from the recent Buncefield JIP $^{1}$. A blast wave diagram was developed ${ }^{11}$ to illustrate typical waves generated during the flame acceleration process and their subsequent interaction. The evaluation showed that the blast wave shape for a deflagration in an elongated congested volume is similar to that for an acoustic wave in the near-field along the long-axis direction. An elongated VCE blast wave has a very quick transition from the positive phase peak pressure to the negative phase peak pressure, relative to the positive phase duration. The magnitude of the applied negative pressure on a building face depends strongly on the transition time between the positive and negative phase 
peak pressures, and this applied negative phase can be important to structural response under certain conditions ${ }^{9}$.

This evaluation extends the previous work $^{11}$, examining how the elongated VCE geometry impacts the resultant blast wave shape in the near-field. The influence of the normalized flame travel distance and the flame speed is also examined.

\section{Terminology}

\subsection{Parameters for Elongated VCEs}

Figure 1 depicts an elongated flammable cloud with a length $\mathrm{L}$, a width $\mathrm{W}$ and a height $\mathrm{H}$. It is assumed that the cloud covers a congested volume (i.e., that none of the cloud occupies an empty uncongested space), so that the cloud and the congested volume have the same dimensions. The parameters shown in Table 2 are utilized to characterize the cloud geometry and the resultant blast field.

Table 2. Cloud Geometry and Blast Field Parameter Definitions

\begin{tabular}{|c|c|c|}
\hline Parameter & Symbol & Definition \\
\hline Free Vent Distance & $\mathrm{L}_{\mathrm{FV}}$ & $\operatorname{MIN}\left(\mathrm{H}, \frac{\mathrm{w}}{2}\right)$ \\
\hline Cylindrical Distance & $\mathrm{L}_{\mathrm{Cyl}}$ & $\operatorname{MAX}\left(\mathrm{H}, \frac{\mathrm{w}}{2}\right)$ \\
\hline Equivalent Radius of Cross Section & $\mathrm{R}_{\mathrm{Eq}}$ & $\sqrt{\frac{2 \cdot \mathrm{H} \cdot \mathrm{W}}{\pi}}$ \\
\hline Flame Travel Distance & $\mathrm{L}_{\mathrm{f}}$ & $\mathrm{n} / \mathrm{a}$ \\
\hline Standoff Distance of Target & $\mathrm{L}_{\mathrm{SD}}$ & $\mathrm{n} / \mathrm{a}$ \\
\hline Normalized Flame Travel Distance & $\mathrm{Ln}_{\mathrm{f}}$ & $\mathrm{L}_{\mathrm{f}} / \mathrm{L}_{\mathrm{FV}}$ \\
\hline Normalized Target Standoff Distance & $\mathrm{Ln}_{\mathrm{SD}}$ & $\mathrm{L}_{\mathrm{SD}} / \mathrm{L}_{\mathrm{FV}}$ \\
\hline
\end{tabular}

The "Cylindrical Distance" $\left(\mathrm{L}_{\mathrm{Cyl}}\right)$ is introduced to measure the maximum distance a cylindrical flame front can travel before reaching the edge of the cloud. The "Flame Travel Distance" $\left(\mathrm{L}_{\mathrm{f}}\right)$ is measured from ignition to the cloud end. The ignition could be located at the cloud center, cloud end or anywhere in the cloud. The "Standoff Distance of Target" $\left(\mathrm{L}_{\mathrm{SD}}\right)$ is the shortest distance measured from the congested cloud boundary to the target of interest. The last two parameters shown in Table 2 are normalized parameters used to characterize flame travel and standoff distance:

For the purposes of this paper, a flammable cloud is considered to be "elongated" if the ratio of the cloud length to the free vent distance $\left(L / L_{F V}\right)$ is larger than 10 and the ratio of the cylindrical distance to the free vent distance $\left(L_{C y l} / L_{F V}\right)$ does not exceed 2. These conditions are 
summarized in Table 3, and ensure that the normalized flame travel distance is at least $\operatorname{Ln}_{\mathrm{f}}=5$ if a cloud is ignited at its center.

The elongated cloud is characterized by two parameters: (1) the aspect ratio $(L / W / H)$ and (2) a characteristic distance represented by either the free vent distance $\left(L_{F V}\right)$ or the equivalent radius $\left(R_{\text {Equiv }}\right)$. The three cloud parameter sets specifically evaluated in previous work ${ }^{11}$ are given in

Table 4. The "Example Case" cloud is a parametric example case, whereas the "Buncefield" and "BakerRisk" clouds correspond to experimental test rigs. The "Example Case" cloud listed in

Table 4 has dimensions of $\mathrm{L}=37 \mathrm{~m}, \mathrm{~W}=7.32 \mathrm{~m}$ and $\mathrm{H}=1.83 \mathrm{~m}$ and has (1) an aspect ratio of $20 / 4 / 1$ and (2) a free vent distance $\left(L_{F V}\right)$ of $1.83 \mathrm{~m}$ and an equivalent radius $\left(R_{\text {Equiv }}\right)$ of $2.92 \mathrm{~m}$ (along with a cylindrical distance of $3.66 \mathrm{~m}$ ). This cloud is referred to as " $20 / 4 / 1-1.83 \mathrm{~m}$ " or "20/4/1 - R 2.92 m" within the context of this paper. The BakerRisk test rig has dimensions of $\mathrm{L}=22 \mathrm{~m}(72 \mathrm{ft}), \mathrm{W}=3.66 \mathrm{~m}(12 \mathrm{ft})$ and $\mathrm{H}=1.83 \mathrm{~m}(6 \mathrm{ft})$ and is referred to as " $12 / 2 / 1-1.83 \mathrm{~m}$ " or "12/2/1 - R 2.06 m". The Buncefield JIP Test3.2 \#6 configuration has dimensions of L=45 m, $\mathrm{W}=4.5 \mathrm{~m}$ and $\mathrm{H}=3 \mathrm{~m}$ is referred to as " $15 / 1.5 / 1-2.25 \mathrm{~m}$ " or " $15 / 1.5 / 1-\mathrm{R} 2.93 \mathrm{~m}$ ".

Table 3. Conditions for Elongated Cloud

\begin{tabular}{|c|c|c|c|}
\hline Condition & Expanded Condition & If $\mathbf{H}>\mathbf{W} / \mathbf{2}$ & If $\mathbf{H}<\mathbf{W} / \mathbf{2}$ \\
\hline $\mathrm{L} / \mathrm{L}_{\mathrm{FV}}>10$ & $\mathrm{~L}>10 \cdot \mathrm{MIN}\left(\mathrm{H}, \frac{\mathrm{W}}{2}\right)$ & $\mathrm{L}>5 \mathrm{~W}$ & $\mathrm{~L}>10 \mathrm{H}$ \\
\hline$L_{C y l} / L_{F V}<2$ & $\mathrm{MAX}\left(\mathrm{H}, \frac{\mathrm{W}}{2}\right) \leq 2 \cdot \mathrm{MIN}\left(\mathrm{H}, \frac{\mathrm{W}}{2}\right)$ & $\mathrm{H}<\mathrm{W}$ & $\mathrm{W}<4 \mathrm{H}$ \\
\hline
\end{tabular}
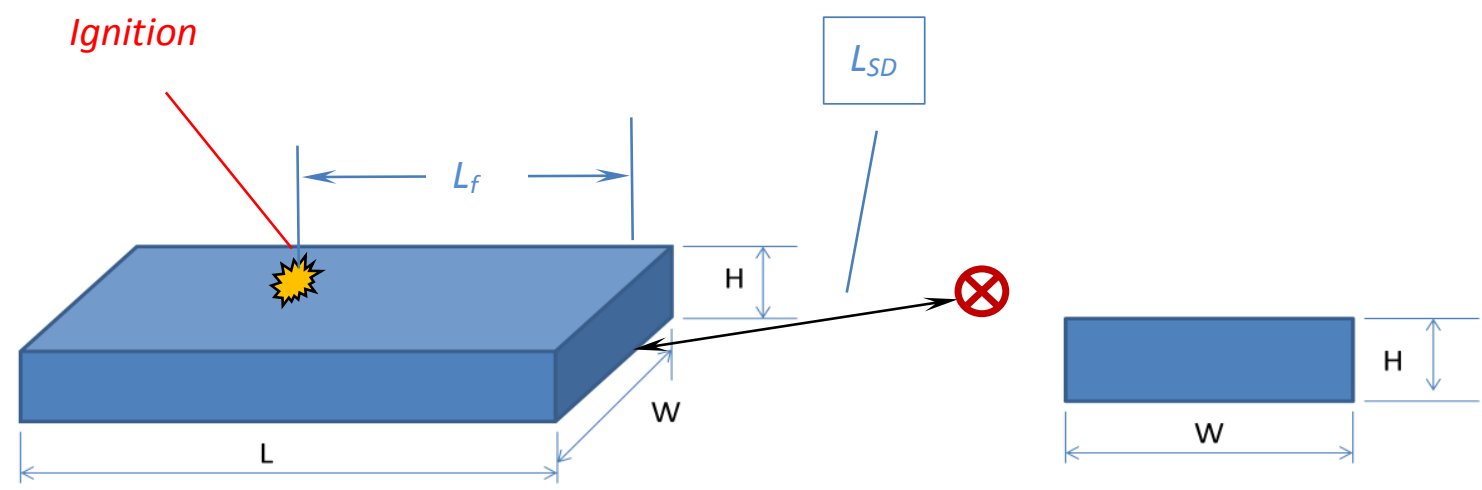
Figure 1. Dimensions of Elongated Vapour Cloud $\left(L / L_{F V}>10\right)$

Table 4. Characteristic Parameters of Elongated Vapour Cloud

\begin{tabular}{|c|c|c|c|c|c|c|c|c|c|}
\hline \multirow{3}{*}{ Test Rig } & \multicolumn{9}{|c|}{ Cloud in Congestion } \\
\hline & \multicolumn{6}{|c|}{ Dimensions [m] } & \multicolumn{2}{|c|}{ Aspect Ratio } & \multirow{2}{*}{$\begin{array}{c}\text { Volume } \\
{\left[\mathbf{m}^{3}\right]}\end{array}$} \\
\hline & \begin{tabular}{|c|} 
Length \\
L
\end{tabular} & $\begin{array}{c}\text { Width } \\
\text { W }\end{array}$ & $\begin{array}{c}\text { Height } \\
\text { H }\end{array}$ & $\mathbf{L}_{-\mathbf{F V}}$ & L_Cyl & $\mathbf{R}_{-}$Equiv & L/W/H - Height & L/W/H - R_Equiv & \\
\hline Example Case & 36.6 & 7.32 & 1.83 & 1.83 & 3.66 & 2.92 & $20 / 4 / 1-1.83 \mathrm{~m}$ & 20/4/1 - R 2.92 m & 490 \\
\hline BakerRisk (12×2x1) & 22.0 & 3.66 & 1.83 & 1.83 & 1.83 & 2.06 & $12 / 2 / 1-1.83 \mathrm{~m}$ & $12 / 2 / 1-\mathrm{R} 2.06 \mathrm{~m}$ & 147 \\
\hline Buncefield JIP (Test3.2 \#6) & 45 & 4.5 & 3 & 2.25 & 3 & 2.93 & $15 / 1.5 / 1-3 \mathrm{~m}$ & $15 / 1.5 / 1$ - R $2.93 \mathrm{~m}$ & 608 \\
\hline
\end{tabular}

\subsection{Characteristic Parameters for Elongated VCEs}

The blast field generated by an elongated cloud depends on several characteristic parameters. The three characteristic parameters examined in this work are as follows:

1) Characteristic time $\left(t_{n}\right)$, which is defined as the time for a rarefaction wave to travel from the cloud edge to the cloud center or ignition location.

2) Energy release rate $(d V / d r)$, which is expressed in terms of the volume of flammable gas cloud consumed per unit flame travel distance ( $V$ is volume and $r$ is the flame front position).

3) Radial expansion factor $\left(F_{E x p}\right)$, which is defined in terms of the expansion of unburned gas ahead of the flame front.

The characteristic time $\left(t_{n}\right)$ is defined by:

$$
t_{n}=\frac{R_{E q}}{C}
$$

where $C$ is the ambient sound speed (taken to be that for air, $334 \mathrm{~m} / \mathrm{s}=1.1 \mathrm{ft} / \mathrm{ms}$ ).

Characterizing the energy release rate as $d V / d R$ assumes a constant energy density in the cloud, which matches the conditions of the test cases evaluated. Of course, $d V / d R$ would actually be the energy release rate only when multiplied by the flame speed and combustion energy released per unit volume. The expression for this parameter depends on the location of the flame front within the cloud. Consider, for example, the case of the "General" cloud ("20/4/1 - 1.83m") with ignition at the cloud center. Figure 2 shows four different flame front positions and corresponding times; expansion and distortion of the cloud due to the passage of the flame front is not reflected in this figure for the sake of simplicity. At time $t_{l}$ the flame reaches the top of cloud (i.e., $\mathrm{r}=L_{F V}$ ), the flame is spherical, and the energy release rate up to this time is:

$$
d V / d r_{r<L_{-F V}}=2 \pi r^{2}
$$


Between $t_{1}$ and $t_{2}$ the flame travels to the side of the cloud/congestion (i.e., at $t_{2}, \mathrm{r}=L_{C y l}$ ), at which point the flame front shape is neither spherical nor cylindrical. The energy release rate during this time period $\left(t_{1}\right.$ to $\left.t_{2}\right)$ can be approximated as:

$$
d V / d r_{L_{F V}<r<L_{C y l}}=2 \pi L_{F V} r
$$

Time $t_{4}$ is defined as that time at which the flame front reaches $2 \mathrm{~L}_{\mathrm{Cyl}}$, and there after the flame front is almost planar and the energy release rate can be expressed as:

$$
d V / d r_{r>2 L_{C y l}}=L W
$$

Between $t_{2}$ and $t_{4}$, the energy release rate can be linearly extrapolated from a rate of $2 \pi L_{-F V} r$ to $L W$.

The radial expansion factor $\left(F_{E x p}\right)$ is a function of flame speed, with a lower flame speed giving a higher expansion factor. The volume expansion factor for gaseous hydrocarbon fuel combustion is around 8 for a stoichiometric mixture with air, which gives a radial expansion factor of between 1 and 2, depending on flame speed.

Figure 3 provides an example of a normalized energy release rate distribution along the long-axis of the cloud, both without and with cloud expansion taken into account, as shown in the top and bottom charts, respectively. The energy release rates were normalized by $L W$, the cloud cross sectional area, so that the energy release rate is unity at long flame travel distances. The flame travel distance was normalized by the free vent distance $\left(L_{F V}\right)$, so that the total cloud length would be approximately 20 (i.e., $37 / 1.83=20.2$ ) and the half-length of the cloud would be approximately 10 . A radial expansion factor $\left(F_{E x p}\right)$ of 1.5 was used for illustrative purposes. The normalized energy release rate distribution without cloud expansion (top chart) matches the schematic description shown in Figure 2. However, the normalized energy release rate distribution with cloud expansion (bottom chart) better explains the blast field resulting from an elongated VCE, as discussed in the following section.

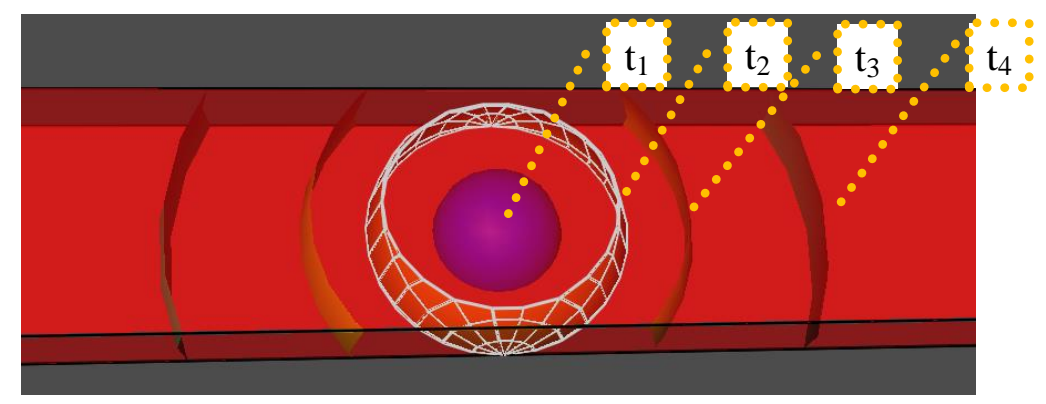

Figure 2. Schematic of Flame Shape in Elongated Cloud (20/4/1 -1.83 m Cloud) 


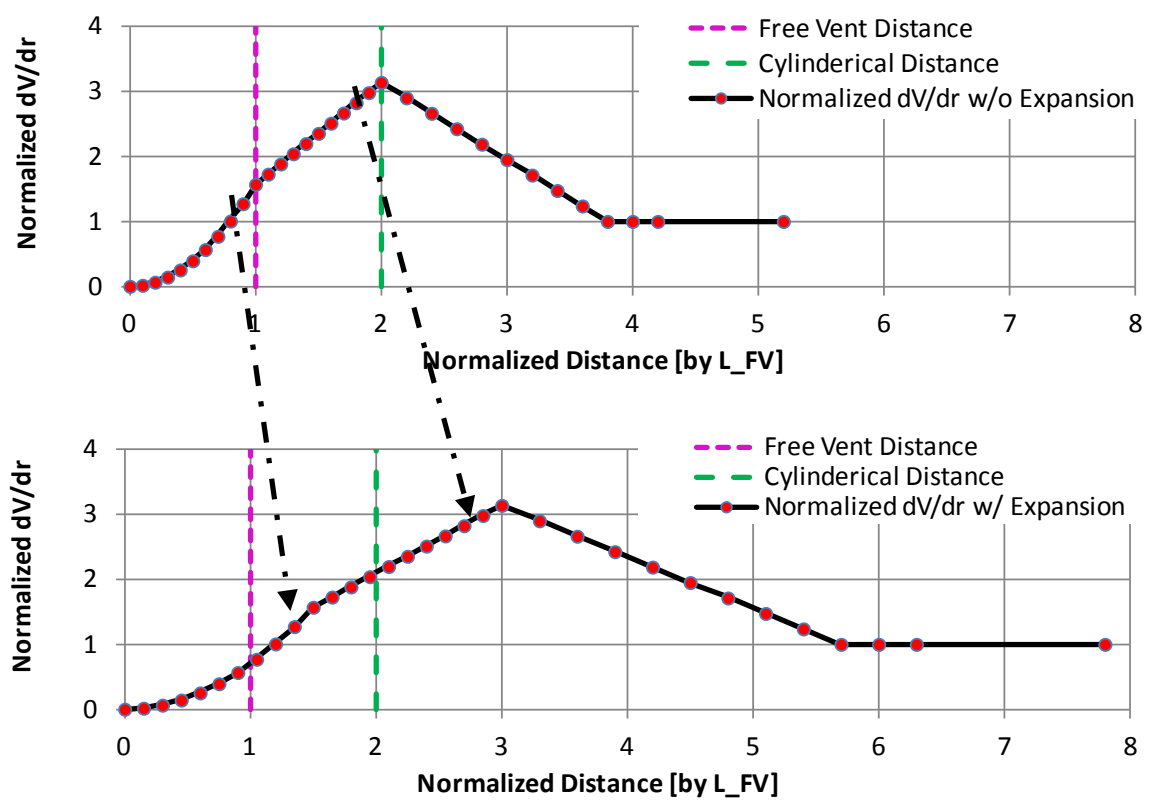

Figure 3. Energy Release Rate Distribution without (top) and with (bottom) Cloud Expansion (20/4/1 -1.83 m Cloud)

\subsection{Example of Elongated VCEs}

A VCE of the "Example Case" cloud (20/4/1 - 1.83 m) configuration, shown in Figure $2 \&$ Figure 3, was numerically modelled using the FLACS CFD $\operatorname{code}^{14,15}$ to illustrate the resulting flammable cloud expansion and blast field. Figure 4 shows the fuel concentration (ER: equivalence ratio) and overpressure contours at selected times. Only half (i.e., the "right half") of the domain is shown in these figures since the cloud was ignited at the center of the cloud at grade level. The fuel ER and pressure scales shown in these figures are from LFL (lower flammable limit) to 1.0 (stoichiometric ER) and from $0.007 \mathrm{barg}(0.1 \mathrm{psig})$ to $0.1 \mathrm{barg}(1.5 \mathrm{psig})$, respectively. The fuel concentration contours are shown to illustrate both the flame front development and the resultant expansion of the unburned fuel cloud. Both elevation (side) and plan (top) views of the fuel concentration contours are provided. The distance scale is normalized distance by the free vent distance $L_{F V}$ (equal to the height of the cloud for this case), so that the cloud half-length is approximately 10 (i.e., only the central portion of the cloud is shown in the fuel concentration contours).

As the flame reaches the initial height of the cloud (i.e., $L_{F V}$ ) at a normalized flame travel distance of 1 and at a time of about 0.26 seconds, the cloud has expanded significantly in the vertical direction near the cloud center, but the expansion along the horizontal direction is a relatively small percentage of the longitudinal length. The pressure continues to build up near the central part of cloud until about $0.26 \mathrm{~s}$ (see Figure 4). As the accelerating flame arrives at the normalized distance of 2 (at around $0.29 \mathrm{~s}$ ), it reaches the sides of the cloud, and the period during which the flame can be characterized as cylindrical ends. A group of rarefaction waves begin traveling inward from the sides as a result, and this continues until a time of about $0.33 \mathrm{~s}$ when the leading flame front reaches a normalized distance of about 4. During this process, a high pressure core moves longitudinally down congestion with the flame front while a negative phase develops in the central portion of the cloud. After $0.33 \mathrm{~s}$, the high pressure core retains the 
same basic pattern as the flame travels through the remainder of the cloud at a nearly constant flame speed, as can be seen in Figure 5, which illustrates the propagation through the remainder of the cloud. Times of $0.37 \mathrm{~s}$ and $0.41 \mathrm{~s}$ correspond to the flame arrival location at the normalized distances of 6 and 8 , respectively.

The flame speed (Mach No.) distribution along the long-axis direction and overpressure histories at selected locations are given in Figure 6 and Figure 7, respectively. A simplified flame speed distribution is also depicted in Figure 6, indicating three phases of the flame acceleration: (1) one during the time when the flame front is spherical, (2) a second when the flame front is cylindrical, and (3) the latter portion of the flame propagation, where the flame speed is approximately steady (constant) when the flame front is planar. These three flame acceleration phases can also be seen in Figure 3. The three distinct energy release rates regimes can be characterized as: (1) $L / L_{F V}$ from 0 to 1.5 , where the flame front is spherical and the flame speed increases in an approximately linear fashion, (2) $L / L_{F V}$ from 1.5 to 3.0, where the flame front is cylindrical and the flame acceleration is reduced, indicating that the energy release by the cylindrical flame front provides a weaker source to support expansion, and (3) $L / L_{F V}$ greater than 3 , where the flame speed reaches approximately Mach 0.27. The drop in the energy release rate in the third regime, as illustrated in Figure 3, can only support a steady flame propagation (with some oscillations about the steady value). The primary pressure peak due to the initial flame acceleration decouples from the flame front as this $3^{\text {rd }}$ regime is entered, which can be seen in the pressure histories at locations beyond $L / L_{F V}=4$, leading to the double peak in the pressure-time history which can be seen in Figure 7.

The double peak pressure signal was also evident in recent tests performed with the "BakerRisk" cloud configuration $(12 / 2 / 1-1.83 \mathrm{~m}$, see

Table 4). The maximum flame speed attained in the tests was $M_{f}=0.4$. The first (or the primary) peak was much more pronounced than the one shown in Figure 7. 


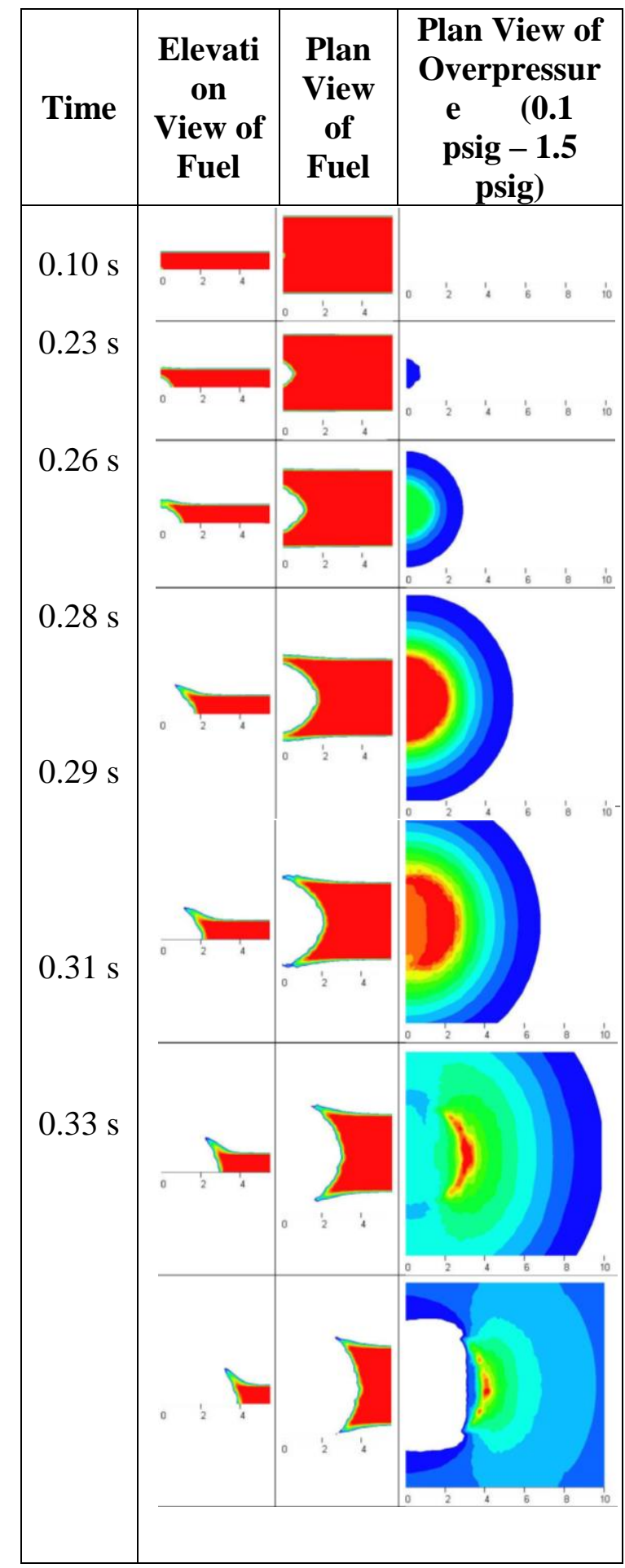

Figure 4. Fuel Concentration and Pressure Contour for an Elongated Vapour Cloud, Example Case (20/4/1 - 1.83 m Cloud, Ctr-Ign, 1 of 2) 


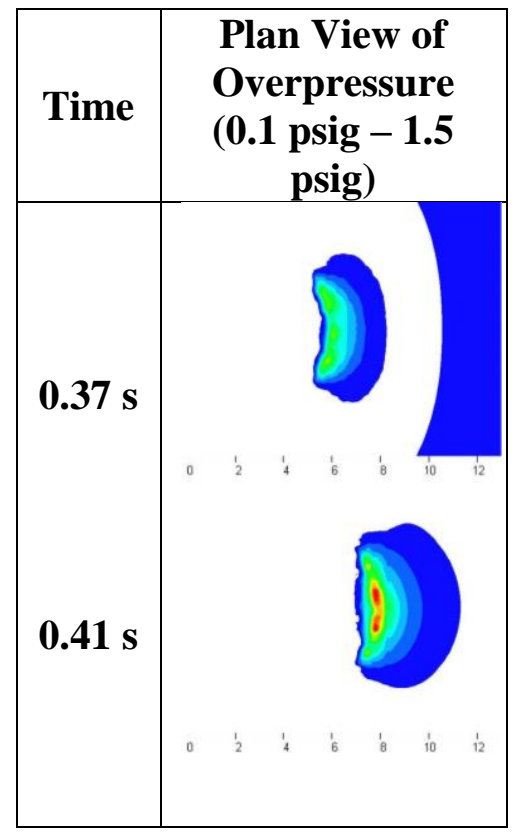

Figure 5. Overpressure Contours with Flame near Cloud End, Example Case (20/4/1 1.83 m Cloud, Ctr-Ign, 2 of 2)

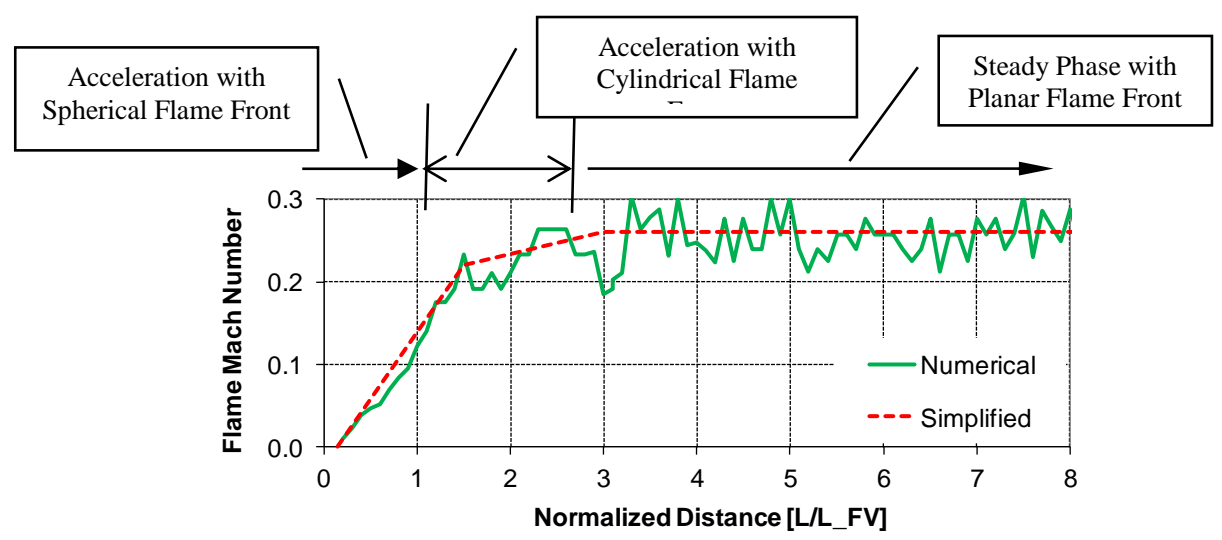

Figure 6. Flame Speed Distribution, Example Case (20/4/1 - 1.83 m Cloud, Ctr-Ign) 


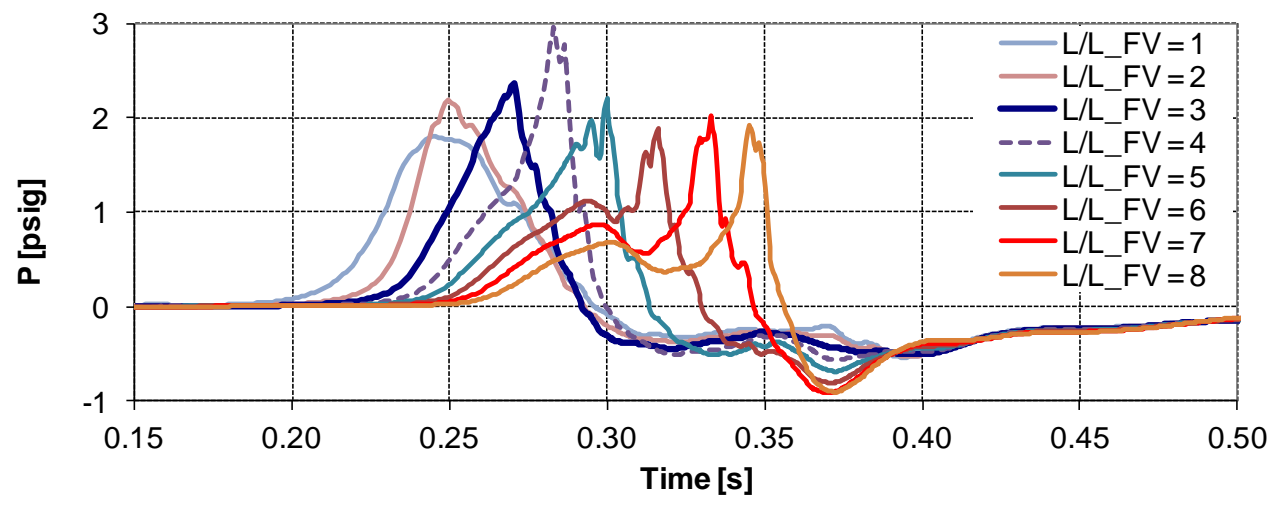

Figure 7. Pressure Histories, Example Case (20/4/1 - 1.83 m Cloud, Ctr-Ign) 2.4 BWTI's Simulation and Validation ${ }^{11}$ against Buncefield JIP Test Data

Table 4 provides the elongated cloud configuration $(15 / 1.5 / 1-2.25 \mathrm{~m}, 15 / 1.5 / 1-\mathrm{R} 2.93 \mathrm{~m})$ for Test 3.2 \#6 from the Buncefield JIP test program. The cloud, which covered a congested volume made up of vegetation, was $45 \mathrm{~m}$ long, $4.5 \mathrm{~m}$ wide and $3 \mathrm{~m}$ high. Ignition was at one end of the cloud, $1 \mathrm{~m}$ inside the cloud edge. The cloud length ratio $\left(L / L_{F V}\right)$ is greater than 15 . BakerRisk's BWTI $^{\text {TM }}$ CFD code was selected to model the blast loads due to the large length ratio. A prescribed flame speed that approximately matched the measured flame speed was used, ensuring that the constant flame speed after the initial acceleration phase in the cloud was modelled reasonably. The blast wave shape predicted by BWTI is in reasonable agreement with the test data, including the double peaks. The agreement for the blast pressure histories at the end of the cloud is particularly good for both the positive and negative peak overpressures, including the transition from the positive phase peak to the negative phase peak ${ }^{11}$.

\section{Characteristics of Elongated VCEs ${ }^{11}$}

For an unconfined elongated VCE with a uniform fuel concentration and congestion pattern, an initial rapid phase of flame acceleration occurs within the normalized distance $\left(L / L_{F V}\right)$ region between 2 and 4. If a deflagration-to-detonation transition (DDT) does not occur within this range, an approximately constant subsonic flame speed will be reached within the normalized distance $\left(L / L_{F V}\right)$ region between 4 and 6 . During the constant flame speed phase, an isolated high pressure core travels with the flame front, followed by a negative phase. The negative phase is generated by the primary blast wave developed during the initial acceleration phase.

The blast wave shape within the elongated cloud and in the near-field exhibits a behavior belonging to the acoustic wave regime no matter how high the flame speed as long as the flame Mach number is less than unity (i.e., subsonic flame), while the magnitudes of the peak positive and negative phases are larger than with a traditional acoustic wave.

At a sufficiently high flame speed $\left(M_{f}>0.6\right)$, the blast wave outside the cloud/congestion (along the long-axis direction) experiences a significant pressure drop from the peak positive pressure to 
peak negative pressure within the normalized characteristic time $\left(t_{n}\right)$. The magnitude of the pressure drop between the peak positive and negative pressures diminishes quickly with distance. For the case where the flame speed reaches Mach 0.9, the pressure at the end of the cloud drops from the peak positive pressure $(9.0 \mathrm{psig})$ to the peak negative pressure $(-10 \mathrm{psig})$ within the normalized characteristic time $\left(t_{n}=8.8 \mathrm{~ms}\right)$. The difference between the peak positive and negative pressure is $19 \mathrm{psig}$ (1.3 barg). A comparable pressure drop occurs at a standoff distance of $6 \mathrm{~m}\left(L n_{S D}=2.7\right)$. The drop between the peak positive and negative pressures diminishes quickly with distance. This localized pressure drop is due to the high pressure core traveling with the flame front (i.e., in the region beyond an $L n_{f}$ of 6) in an elongated VCE.

Note these observations were made with the congested volume of $15 / 1.5 / 1-2.25 \mathrm{~m}$ or $15 / 1.5 / 1-$ $\mathrm{R} 2.93 \mathrm{~m}$.

\section{Deflagration versus DDT Regime}

The previous work ${ }^{11}$ also concluded that if the flame speed does not exceed Mach 0.6 (i.e., $M_{f}$ $<0.6)$ within a normalized distance $\left(L / L_{F V}\right)$ of 6 , the slow speed of the steady flame allows the primary and second pressure waves to decouple beyond a normalized distance of approximately 6 , resulting in the rapid decay of the first peak, while the second peak remains at a constant value (depending on the steady flame speed achived).

If a flame speed of Mach 0.9 is attained within a normalized distance $\left(L / L_{F V}\right)$ of 2 and 4 , the decoupling between the primary peak and the secondary peak beyond an $L / L_{F V}$ of 4 is diminished. A DDT may occur if a positive-feedback disturbance (e.g., congestion pattern change) within the normalized distance range of 4 to 6 is present.

At a flame speed of between Mach 0.6 and 0.9, the decoupling between the primary and secondary peaks is not significant in the normalized distance $\left(L / L_{F V}\right)$ region between 4 and 6 , and hence flames traveling at this speed would be most susceptible to a DDT in this range. Of course, the disturbance required for a Mach 0.6 flame to DDT would be much greater than with a Mach 0.9 flame.

Figure 8 illustrates deflagration versus DDT regimes as a function of normalized distance and flame speed based on these observations. DDTs in BakerRisk unconfined test rig were reported for both ethylene ${ }^{16,17}$ and hydrogen ${ }^{18}$ mixtures, as shown in Figure 9 (a). A DDT resulted for ethylene within a normalized distance $\left(L / L_{F V}\right)$ of 4 for an approximately stoichiometric mixture. With a hydrogen mole fraction of less than 18\%, no DDT was observed and the deflagration flame speed was less than Mach 0.6. With a hydrogen mole fraction of about $22 \%$, a DDT occurred within a normalized distance $\left(L / L_{F V}\right)$ of around 3.

Figure 9 (b) was generated based on the Buncefield JIP test data ${ }^{1}$. Propane-air mixtures with an approximately stoichiometric concentration were used for all eight tests, with changing congestion patterns in terms of congestion levels and free vent distances $\left(L_{F V}\right)$. Two DDT data sets and one deflagration data set are shown in Figure 9 (b). With the worst congestion pattern (3 trees $/ \mathrm{m}^{2}, L_{F V}=2.25 \mathrm{~m}$ ), a DDT occurred within a normalized distance $\left(L / L_{F V}\right)$ of 4 . With a moderate congestion pattern $\left(2\right.$ trees $\left./ \mathrm{m}^{2}, L_{F V}=2.25 \mathrm{~m}\right)$ a DDT occurred within a normalized distance $\left(L / L_{F V}\right)$ of around 6. With a less severe congestion pattern $\left(1.5\right.$ trees $\left./ \mathrm{m}^{2}, L_{F V}=2.25 \mathrm{~m}\right)$, 
no DDT was observed, resulting in a deflagration with a flame speed less than Mach 0.6.

The corresponding regimes are in general agreement with the observed DDTs and deflagrations in unconfined vapor clouds discussed above.

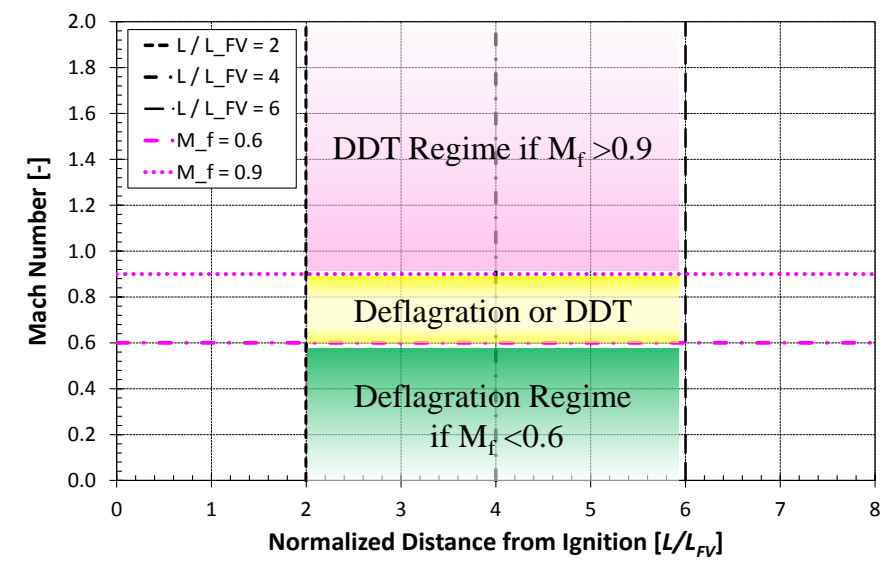

Figure 8. Deflagration vs. DDT Regimes

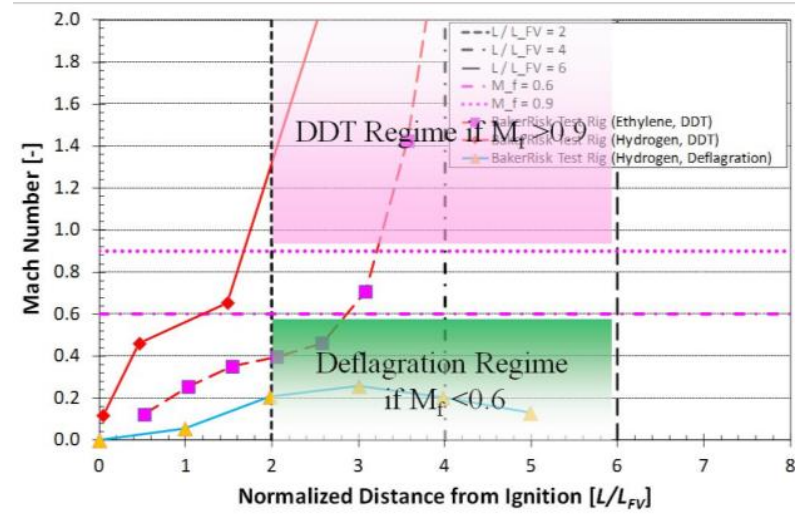

(a) BakerRisk Test Rig

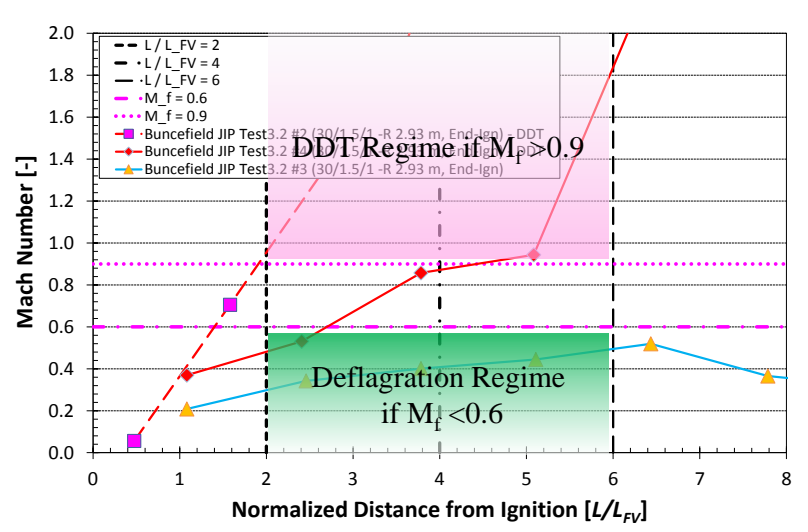

(b) Buncefield JIP Test Rig

Figure 9. Observed Test Results

\section{Near-field Blast Wave Shape}

BakerRisk's BWTI code was further used to perform the parametric study described in this section to investigate blast wave shape differences in the far-field as a function of flame travel distance and flame speed attained at the steady phase. The initial flame acceleration was assumed to occur within a normalized distance of 3 .

\subsection{Typical Blast Wave Shapes in Near-field around Elongated Cloud}

Figure 10 shows a BWTI model with an aspect ratio of 12/1/1 - 3.0 m. Targets are distributed along the long-axis direction (Targets 1 through 25), 45 degrees from the cloud end (Targets 26 through 35), the short-axis direction from the cloud end (Targets 36 through 44), and the shortaxis direction from the cloud center (Targets 45 through 50). Pressure histories at locations around the cloud boundary with a normalized standoff distance of about 3 are given in Figure 10. 
The cloud is centrally ignited and the flame speed attained at a steady state is $M_{f}=0.6$. It is clearly seen that the blast wave shape at Target 46 (i.e., along short-axis direction from the cloud center) exhibits a centrally peaked wave characteristic of a standard VCE (i.e., a hemispherical cloud). The blast wave front is steeper at Target 36 (i.e., along short-axis direction from the cloud end), but still has a typical VCE blast wave shape. At Target 27 along the 45 degree direction from the cloud end, the later part of the positive phase is terminated by a sharp pressure decrease to the negative phase. This trend is enhanced at Target 19 along the long-axis direction from the cloud end, with two distinct positive pressure peaks and a very pronounced negative phase peak.

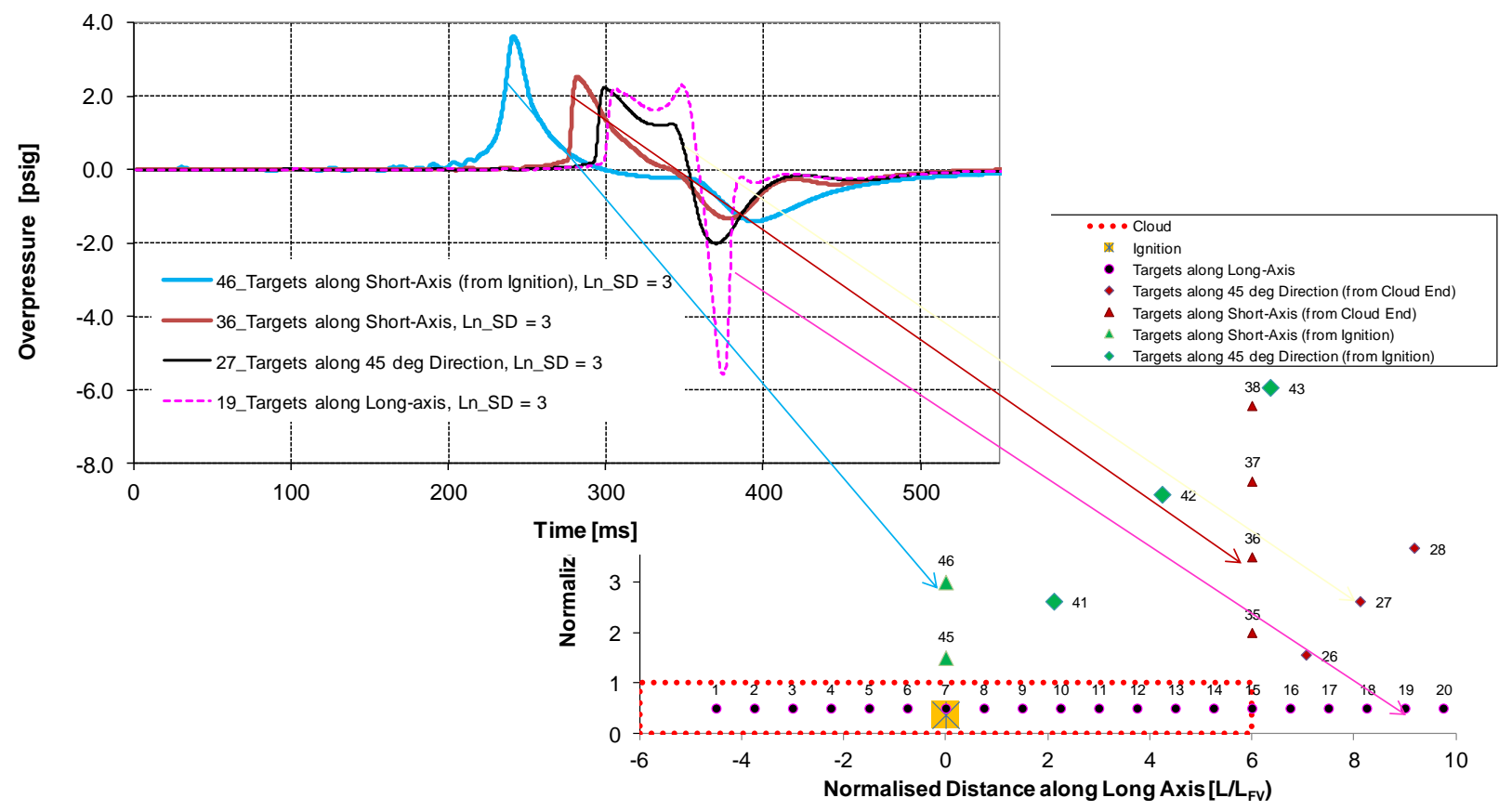

Figure 10. Near-field Blast Waves at Selected Locations $\left(\operatorname{Ln}_{\mathrm{f}}=6 ; M_{\mathrm{f}}=0.6\right)$

\subsection{Impact of Flame Travel Distance}

Two normalized flame travel distances $\left(\operatorname{Ln}_{\mathrm{f}}=6 \& 11\right)$ were examined for a flame with a steadystate flame speed of Mach 0.6. The corresponding pressure histories at a normalized distance of 3 are given in Figure 11. There is no significant difference in between these cases for the blast wave positive phase at Target 46. However, there are significant differences between the two cases at targets near the end of the elongated cloud (i.e., Targets 36, $27 \& 19$ ). There is a lower first positive pressure peak and a longer duration for the case of $\operatorname{Ln}_{\mathrm{f}}=11$ than those for the case of $\operatorname{Ln}_{\mathrm{f}}=6$, while the second positive pressure peak remains essentially unaffected. 


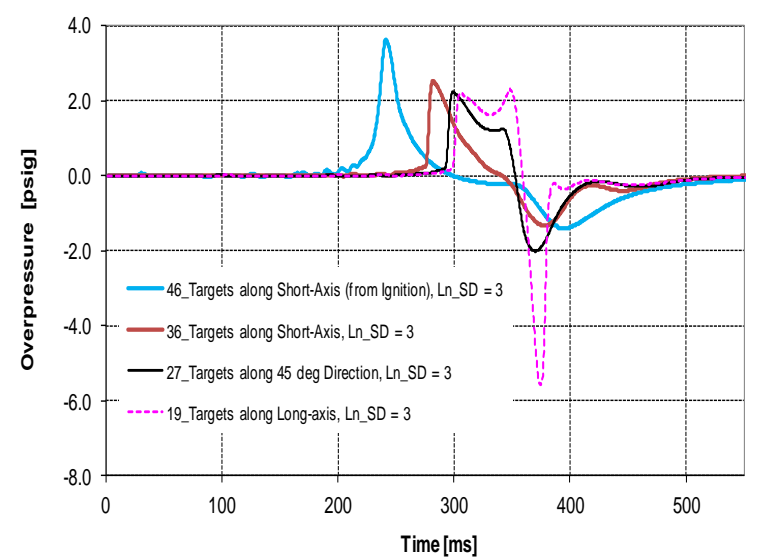

$\operatorname{Ln}_{\mathrm{f}}=6$

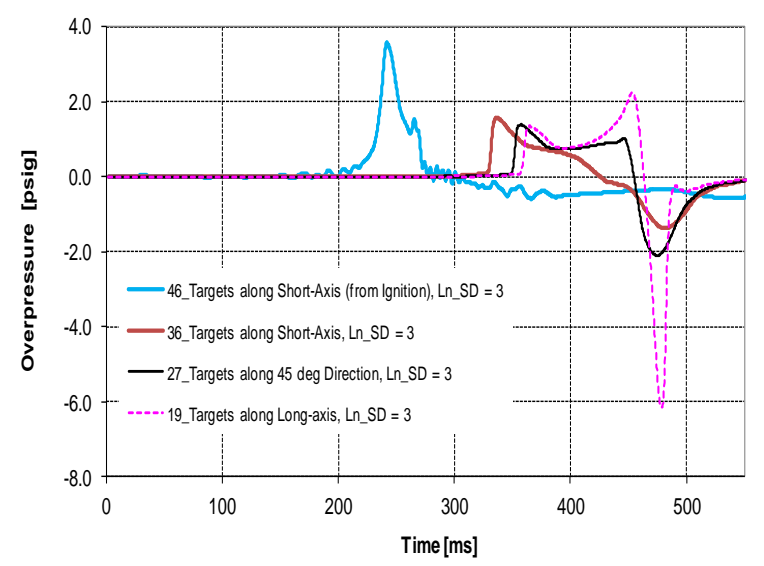

$\mathrm{Ln}_{\mathrm{f}}=11$

Figure 11. Influence of Flame Travel Distance on the Blast Wave Shape $\left(M_{f}=0.6\right)$

\subsection{Impact of Flame Speed}

Figure 12 shows the impact of the flame speed on the resultant near-field blast pressure histories. Two flame speeds $\left(M_{f}=0.5 \& 0.7\right)$ were examined for a fixed congested cloud size $\left(\operatorname{Ln}_{\mathrm{f}}=11\right)$. The pressure histories at a normalized distance of 3 are given in Figure 12. With the higher flame speed, the magnitudes of both the second positive pressure peak and the negative pressure peak at near-field targets along the long-axis direction are larger, with a shorter positive duration. The time from the second positive pressure peak to the negative peak is almost the same (i.e., equal to about one normalized time, $t_{n}$ ).

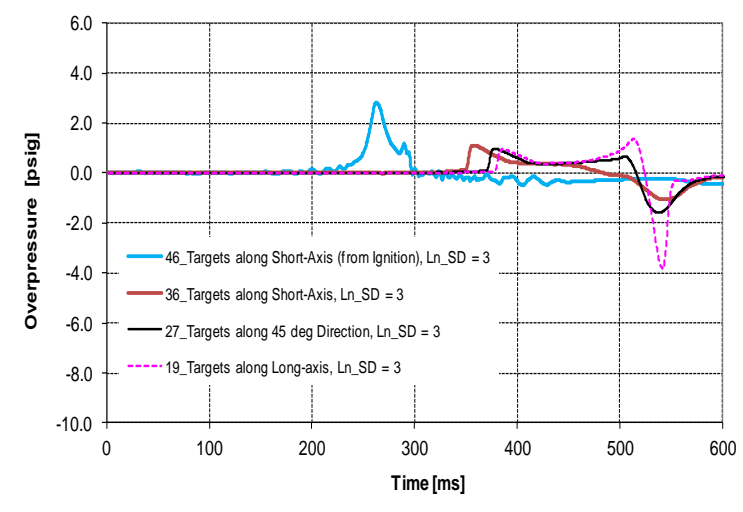

$M_{f}=0.5$

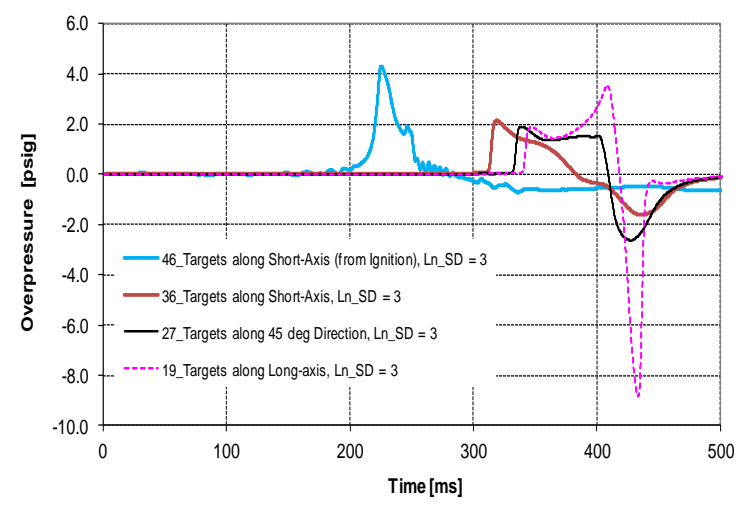

$M_{f}=0.7$

(a) Figure 12. Influence of Flame Speed on the Blast Wave Shape $\left(\operatorname{Ln}_{\mathrm{f}}=11\right)$

\section{Conclusions}

The following conclusions were reached as a result of this evaluation and the previous work ${ }^{11}$ :

- For an unconfined elongated VCE with a uniform fuel concentration and congestion pattern, an initial rapid phase of flame acceleration occurs within the normalized distance $\left(L / L_{F V}\right)$ region between 2 and 4 . An approximately constant subsonic flame speed will be reached within the normalized distance $\left(L / L_{F V}\right)$ region between 4 and 6 if a DDT does not 
occur,. During the constant flame speed phase, an isolated high pressure core travels with the flame front, followed by a negative phase. The negative phase is generated by the primary blast wave developed during the initial acceleration phase.

- For the case with a higher flame speed $\left(M_{f}>0.6\right)$, the blast wave outside the cloud/congestion experiences a significant pressure drop from the peak positive pressure to peak negative pressure within the normalized characteristic time $\left(t_{n}\right)$. The magnitude of the pressure drop between the peak positive and negative pressures diminishes quickly with distance.

- The blast wave shape within the elongated cloud and in the near-field along the long-axis exhibits a behavior belonging to the acoustic wave regime as long as the flame Mach number is less than unity (i.e., subsonic flame), while the magnitudes of the peak positive and negative phases are larger than with a traditional acoustic wave.

- If a flame speed of Mach 0.9 is attained within a normalized distance $\left(L / L_{F V}\right)$ of 2 and 4, the decoupling between the primary peak and the secondary peak beyond an $L / L_{F V}$ of 4 is diminished. A DDT may occur if a positive-feedback disturbance (e.g., congestion pattern change) within the normalized distance range of 4 to 6 is present.

- The identified deflagration and DDT regimes for unconfined elongated VCEs as a function of normalized flame travel distance $\left(L n_{f}\right)$ and flame speed attained at a specified $L n_{f}$ were found to be in reasonable agreement with published data.

- The blast wave shape in near-field around the congested cloud boundary can be significantly impact by the cloud aspect ratio and flame speed attained.

Further study is required to investigate the impact of the flame acceleration distance on the resultant blast wave shapes, particularly in the near-field along the long-axis directions.

\section{References}

1) B. A. Burgan, Dispersion and Explosion Characteristics of Large Vapour Clouds (Volume 2 - Appendices), SCI DOCUMENT ED024, 2014

2) Baker, W.E., Explosion in Air, University of Texas Press, Austin, TX, 1973.

3) Center for Chemical Process Safety, Guidelines for Vapor Cloud Explosion, Pressure Vessel Burst, BLEVE and Flash Fire Hazards, American Institute of Chemical Engineers, John Wiley \& Sons, Hoboken, NJ, 2010.

4) Strehlow, R.A., Luckritz, R.T., Adamczyk, A.A. and Shimp, S.A.,"The Blast Wave Generated By Spherical Flames", COMBUSTION AND FLAME, 35: 297-310, 1979

5) Baker, W.E., Cox, P.A., Westine, P.S., Kulesz, J.J. and Strehlow, R.A., Explosion Hazards and Evaluation, Elsevier, 1983.

6) van den Berg, A.C., "The Multi-Energy Method - A Framework For Vapor Cloud Explosion Blast Prediction", J. of Hazard Materials, 12, 1-10, 1985

7) Lenoir, E.M., and Davenport, J.A., "A Survey of Vapor Cloud Explosions-Second Update”, $26^{\text {th }}$ Loss Prevention Symposium, AIChE, 1992

8) Tang, M.J. and Baker, Q.A., "A New Set of Blast Curves for Vapor Cloud Explosions," Center for Chemical Process Safety (CCPS)/AIChE, $33^{\text {rd }}$ Loss Prevention Symposium, 1999. 
9) Geng, J., Mader, T. and Baker, Q.A., "Blast Wave Clearing Behavior for Positive and Negative Phases" J. of Loss Prevention in the Process Industries 37: 143-151. 2015

10) Pickles, J.H. and Bittleston, S,H., "Unconfined Valor Cloud Explosions - The Asymmetrical Blast from an Elongated Cloud", COMBUSTION AND FLAME, 51: 45-53, 1983

11) Geng, J., Thomas, J.K., T. and Baker, Q.A., "Characteristics of Elongated Vapour Cloud Explosions" Hazards 25-126, Edinburgh, May 13-15, 2015

12) Geng, J. and Thomas, J.K., "Simulation and Application of Blast Wave-Target Interaction," Proceedings of the 41st Annual Loss Prevention Symposium, Houston, Texas, 2007.

13) Geng, J., Thomas, J.K. and Baker, Q.A., "Pressure Vessel Burst Directional Blast Effects," Paper No. PVP2011-57167, ASME 2011 Pressure Vessels and Piping Conference, Baltimore, MD, July 17-21, 2011.

14) Bakke, J.R., Bjerketvedt, D. \& Bjørkhaug, M. "FLACS as a Tool for Safe Design against Accidental Gas Explosions". IChemE Conference 'Piper Alpha-Lossons for Life Cycle Safety Management'. 26-27 September 1990, London: 12 pp, 1990.

15) van Wingerden, K., Arntzen, B., Storvik, I., Teigland, R., Bakke, J.R., Sand, I.Ø. \& Sørheim, H.R. "FLACS-93: A New Explosion Simulator". ERA-Conference: Offshore Structural Design against Extreme Loads, 3-4 November, London, UK: 5.2.1-5.2.14, 1993.

16) Thomas, J.K., A.J. Pierorazio, M. Goodrich, M. Kolbe, Q.A. Baker and D.E. Ketchum (2003) "Deflagration to Detonation Transition in Unconfined Vapor Cloud Explosions," Center for Chemical Process Safety (CCPS) 18th Annual International Conference \& Workshop, Scottsdale, AZ, 23-25 September 2003.

17) Thomas, J.K., M.L. Goodrich and R.J. Duran (2013) "Propagation of a Vapor Cloud Detonation from a Congested Area into an Uncongested Area: Demonstration Test and Impact on Blast Load Prediction," Process Safety Progress, 32(2): 199-206

18) Thomas, J.K., R.J. Duran and M.L. Goodrich (2010) "Deflagration to Detonation Transition in a Lean Hydrogen-Air Unconfined Vapor Cloud Explosion," Mary Kay O'Connor Process Safety International Symposium," College Station, TX, October 27, 2010. 